\title{
Les zones d'activités à la périphérie des agglomérations vosgiennes
}

Étude comparée d'Épinal et de Saint-Dié

Activity zones at the periphery of Vosgian cities. A comparative study of Epinal and Saint-Dié

Die Gewerbeparks am Rand der Städte der Vogesen : vergleichende

Untersuchung von Épinal und Saint Dié

\section{Colette Renard-Grandmontagne}

\section{(2) OpenEdition}

\section{Journals}

Édition électronique

URL : http://journals.openedition.org/rge/2390

ISSN : 2108-6478

Éditeur

Association des géographes de l'Est

Édition imprimée

Date de publication : 1 juin 2003

ISSN : 0035-3213

Référence électronique

Colette Renard-Grandmontagne, « Les zones d'activités à la périphérie des agglomérations

vosgiennes ", Revue Géographique de l'Est [En ligne], vol 43 / 3 | 2003, mis en ligne le 27 novembre 2010, consulté le 07 septembre 2020. URL : http://journals.openedition.org/rge/2390

Ce document a été généré automatiquement le 7 septembre 2020

Tous droits réservés 


\section{Les zones d'activités à la périphérie des agglomérations vosgiennes}

Étude comparée d'Épinal et de Saint-Dié

Activity zones at the periphery of Vosgian cities. A comparative study of Epinal and Saint-Dié

Die Gewerbeparks am Rand der Städte der Vogesen : vergleichende

Untersuchung von Épinal und Saint Dié

Colette Renard-Grandmontagne

1 Troisième département lorrain par sa population, les Vosges comptaient 380952 habitants au recensement de 1999. Si les deux agglomérations ${ }^{1}$ les plus importantes, Épinal (62 504 habitants) et Saint-Dié (29 443 h) ne représentent qu'un peu moins du quart de cette population, elles concentrent près de la moitié de la superficie des zones d'activités du département. Celles-ci sont presque totalement concentrées dans les deux grandes vallées de la Moselle et de la Meurthe, c'est-à-dire dans les deux anciens couloirs du textile. La carte de la figure 1 (hors texte, dans le cahier couleur) souligne le caractère très ponctuel des zones d'activités dans la partie occidentale, rurale, du département. "Zone industrielle », "lotissement industriel », "zone d'activités ", " parc économique»: les expressions sont multiples pour désigner ces espaces, bien signalisés en bordure des axes routiers et des entrées de ville, et qui regroupent, sur une dizaine d'hectares pour les plus petits ou une centaine pour les plus vastes, des établissements industriels, commerciaux, de transport et de logistique ${ }^{2}$. Cette carte ne recense pas seulement les zones qui ont fait l'objet d'une procédure d'aménagement, mais aussi les zones spontanées regroupant plusieurs établissements et qui sont identifiées, reconnues par la signalétique. 
Figure 1 : Les principales zones d'activités vosgiennes - création et superficie.

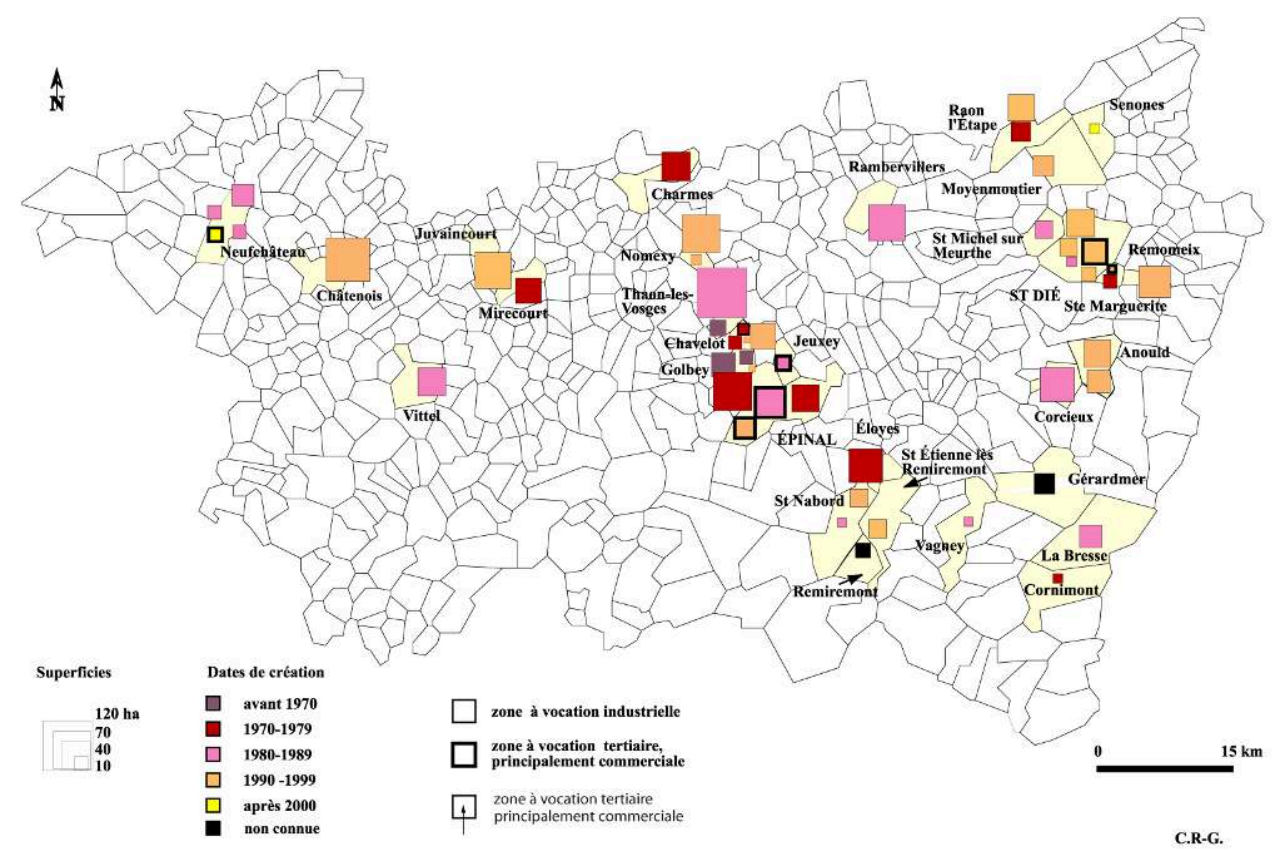

Situées en lisière externe des communes-centres, sur le territoire des communes de banlieue ou plus loin encore, à la périphérie des agglomérations, ces zones d'activités apparaissent comme des excroissances contemporaines (moins de cinquante ans, pour les plus anciennes) de chaque ville. Le sont-elles réellement? Qui est à l'origine de ces créations? Quel rôle jouent les entreprises dans ces développements spatiaux éclatés de la ville? Les exemples de ces deux agglomérations moyennes vosgiennes devraient permettre d'apporter quelques éléments de réponse à ces interrogations et de nourrir une comparaison. Une observation même superficielle met en évidence la grande similitude de certaines de ces zones, en particulier celles à vocation commerciale. Les différences repérées entre les ZI, aussi bien pour ce qui est de leur situation, de leur morphologie, de leur occupation que de leur genèse requièrent une étude plus précise.

Les zones industrielles (ZI) constituent la forme première des zones d'activités. C'est à Golbey que se trouve la plus ancienne du département (Golbey 1, 1958). C'est pourquoi nous commencerons par l'étude des ZI de l'agglomération spinalienne.

\section{I. Épinal : un développement ancien des ZI au nord de la Cite des images}

\section{Une sécrétion spinalienne?}

Canalisées par la vallée de la Moselle, les ZI de l'agglomération spinalienne s'étirent, sur une quinzaine de kilomètres, du nord de la ville jusqu'à Nomexy, le plus souvent sur la rive gauche de la rivière. La carte figure $2 a$ (hors texte, dans le cahier couleur) semble indiquer une corrélation entre distance de la ville-centre et date de création: les plus anciennes étant les plus proches (Golbey 1 et 2), les plus récentes se localisant le plus loin (Nomexy) $)^{3}$. Cette pulvérisation directionnelle à rayon d'action de plus en plus grand semble être le résultat d'une action concertée et programmée dans le temps. 
Programmée par qui? La ville-centre, Épinal? Une structure administrative coordinatrice telle qu'un syndicat intercommunal ? La consultation du tableau 1, en particulier celle des deux dernières colonnes, indique déjà que cet agglomérat linéaire de zones est plus le fruit d'initiatives locales, communales, que d'actions concertées.

Figure 2 : a. Les zones d'activités de l'agglomération d'Epinal - création. b. Les zones d'activités de l'agglomération de Saint-Dié - création

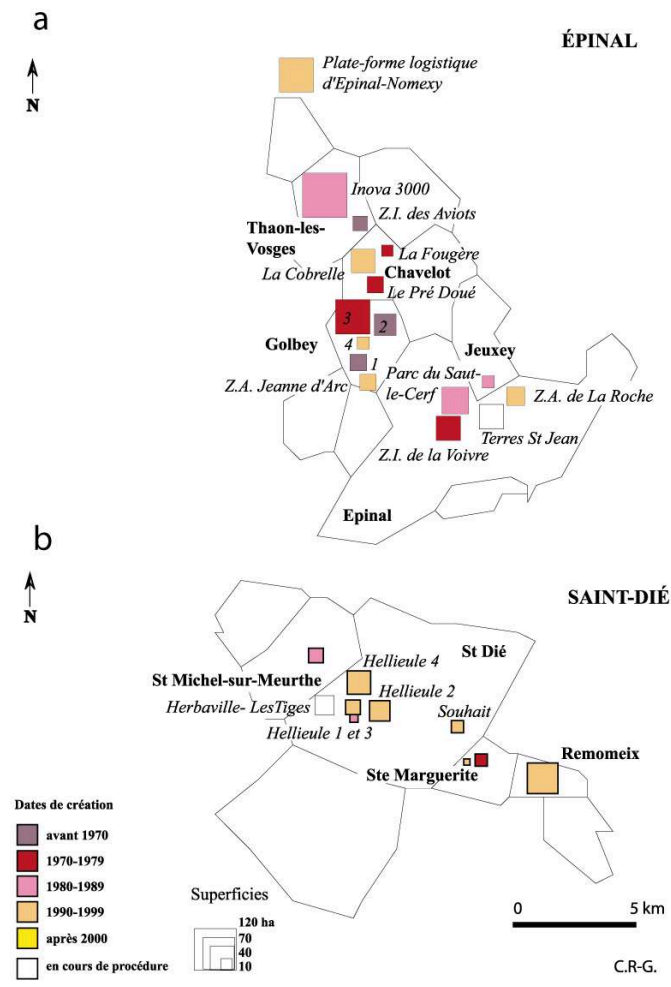


Tableau 1 : Les zones d'activités dans l'agglomération d'Épinal (situation 2003)

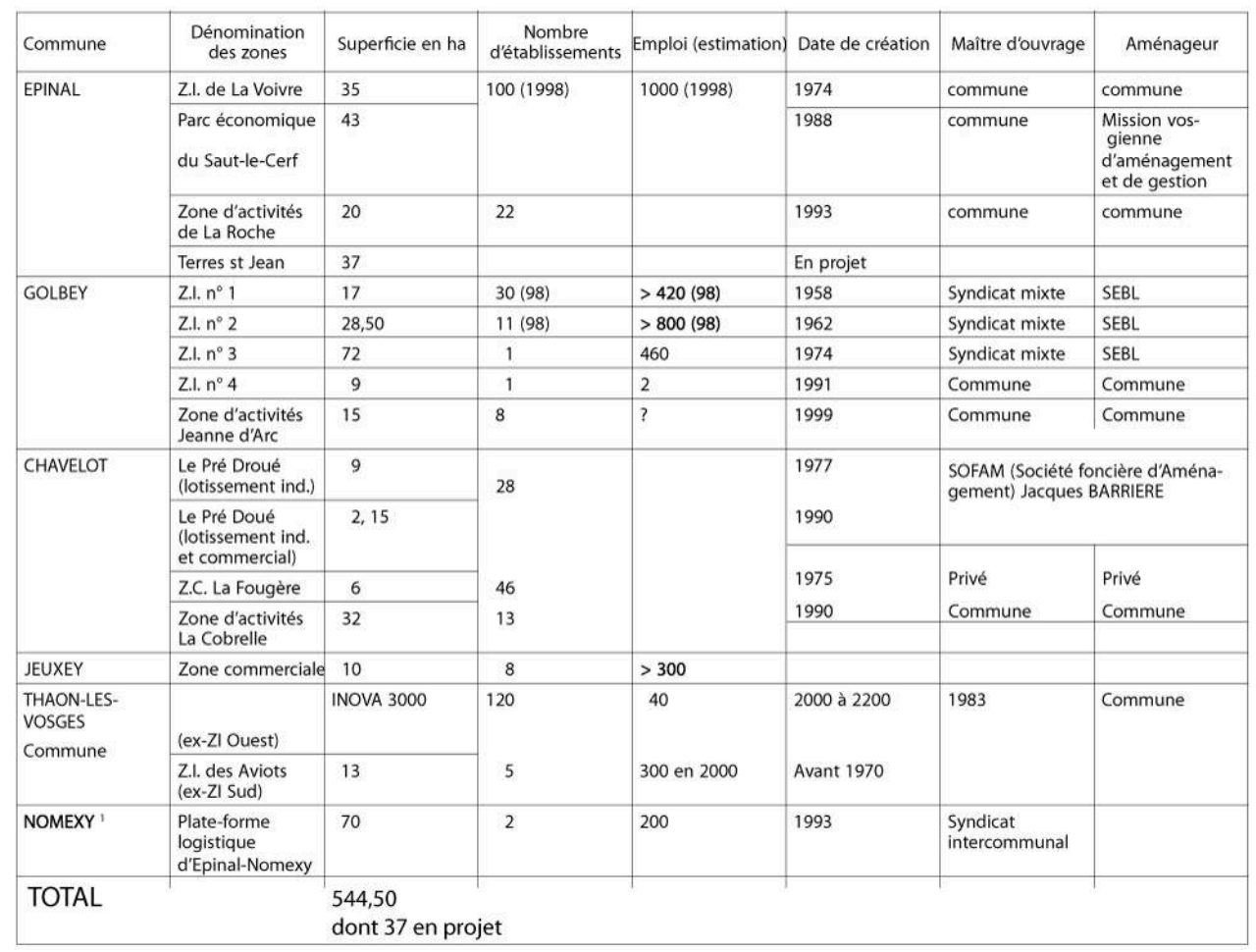

1. Nomexy n'appartient pas à l'agglomération d'Epinal définie par l'INSEE, en 1999

Tableau réalisé d'après enquêtes personnelles et:

- L'industriel. Répertoire des sites industriels : Lorraine 1992, Les Editions L'Industriel, 1992

- CAPEV et Région LORRAINE, Plaquettes de présentation de zones (1992)

- CAPEV / VALORIS, carte des zones industrielles équipées dans les Vosges, 2001

- capev@wanadoo.fr, nouvelle présentation numérisée des zones d'activités (2003)

- DDE des Vosges, Service de l'Urbanisme. Les zones d'activités des Vosges - Situation actuelle Perspectives, janvier 1993

- DDE des Vosges, Service Urbanisme et Aménagement, Cellule Etudes Générales, Situation actuelle des zones d'activités dans le département des Vosges, pour l'aménagement et le développement du territoire, avril 1998

- DDE des Vosges, Service Urbanisme et Aménagement, Cellule documents d'urbanisme, Situation des documents d'urbanisme au ler janvier 2001.

5 Sur les onze ZI, seules quatre (soit environ le tiers) sont nées d'un syndicat mixte (structure coordinatrice). Ces syndicats sont des structures à géométrie variable en fonction des projets. C'est ainsi que le syndicat mixte qui fut le maître d'ouvrage pour la réalisation des ZI de Golbey 2 et 3, a été créé en 1961 et comprenait le département des Vosges, la CCI d'Épinal, la ville d'Épinal, ainsi que celle de Golbey à partir de 1969 et, enfin, la commune de Chavelot à partir de 19784. Néanmoins, en 1999, à l'occasion de l'installation de la deuxième machine à papier aux papeteries de Golbey (zone 3), dans les nouveaux bâtiments dont l'emplacement avait été prévu sur le plan initial, fut constituée une communauté de communes de deux membres (Épinal et Golbey). Cette communauté de communes créait une ZAE (Zone d'Action Économique) intercommunale dont la surface coïncidait exactement avec la superficie nécessaire à cette extension. Cette création de ZAE intercommunale, sur laquelle est perçue une TPZ (Taxe Professionnelle de Zone) d'un taux de 14,84\%, en 2003, a constitué, pour ces collectivités locales, un moyen de ne pas perdre le bénéfice de la manne financière apportée par la mise en route de la deuxième machine. En effet, le code général des 
impôts prévoit un plafonnement de la taxe professionnelle perçue par une commune. Au-delà de la base d'écrêtement, les sommes dues au titre de la taxe professionnelle sont versées au fonds de péréquation départemental.

Dans le cas de la zone industrielle d'Épinal-Nomexy, c'est un autre montage et d'autres motivations qui ont présidé à la création de la ZI. En 1992, en effet, fut formé un syndicat intercommunal Épinal-Nomexy en vue d'aménager la plus grande zone industrielle du département des Vosges (60 à 70 hectares), susceptible d'accueillir un grand établissement industriel (Mercédès, par exemple). Ce projet est né de la rencontre de deux besoins mais aussi de contraintes : la nécessité pour la commune de Nomexy, affectée par la crise du textile, de créer des emplois, et le besoin pour la commune d'Épinal, asphyxiée par le manque de terrains, de se doter de nouvelles surfaces disponibles à l'extérieur de son territoire, en fonction des alliances possibles. La réalisation de cette zone, le long de la voie rapide, en dehors de l'agglomération, est l'exemple d'un mariage de raison ${ }^{5}$ entre une commune qui avait de l'espace disponible mais pas les moyens financiers suffisants pour l'aménager et une seconde commune sans réserve foncière appropriée. Mariage de raison, certes, mais pas réellement mariage de proximité car d'autres réalisations concurrentes s'étaient déjà intercalées entre les deux localités.

7 Toutes ces zones nées d'un syndicat mixte ou intercommunal furent aménagées par la SEBL (Société d'Équipement du Bassin Lorrain), société régionale d'économie mixte créée en 1956.

Les autres zones industrielles sont d'origine « individuelle ", c'est-à-dire communales tant pour la maîtrise d'ouvrage que pour l'aménagement. L'exemple de la zone INOVA 3000 de Thaon-les-Vosges est représentatif à cet égard. Alors que l'activité industrielle de la ville était traditionnellement centrée sur l'espace de la BTT (Blanchisserie et Teinturerie de Thaon), entre Moselle et canal de l'Est, c'est maintenant dans la partie occidentale de la commune que l'essentiel de cette activité réside, le long de la voie rapide Nancy-Remiremont. Cette zone (ex ZI-Ouest de Thaon) s'est développée progressivement en trois extensions successives depuis 1983, autour de l'établissement américain Garrett (devenu Allied Signal Turbo SA, aujourd'hui Garrett-Honeywell) implanté en 1979. Par l'achat de terrains aux particuliers, soit à l'amiable, soit par expropriation, la superficie de la zone est passée de 40 à 120 hectares. Elle constitue la zone la plus vaste, d'un seul tenant, du département.

Née, somme toute, du cumul d'initiatives locales, parfois en partenariat avec une autre commune, cette offre de terrains industriels est-elle adaptée à la demande ? Génère-telle une demande?

\section{Des réponses aux demandes d'entreprises}

10 L'examen des établissements installés dans ces zones fait apparaître que dans chacune des principales zones industrielles, il existe un établissement majeur en superficie et en effectifs. Ce sont, la Trane (société américaine fabriquant du matériel de climatisation et de chauffage) à Golbey 1, Michelin à Golbey 2, les papeteries Norske Skog Golbey qui occupent seules l'intégralité de la zone de Golbey 3, Total Gaz à Golbey 4, les Magasins Généraux d'Épinal dans la zone d'activités de la Cobrelle, à Chavelot, Garrett à Thaonles-Vosges, enfin. Seule la zone d'Épinal-Nomexy est encore peu occupée. 
11 Ce constat signifierait-il que ces zones ont été aménagées à la demande d'industriels ? La demande aurait-elle précédé l'offre? La consultation d'études relatant en partie l'histoire de ces zones et la rencontre d'élus locaux ou de responsables territoriaux peuvent le laisser penser. Si l'aménagement matériel de la zone de Golbey 2 a été accéléré par l'annonce de l'implantation quasi-immédiate de la manufacture Michelin (été 1968), si la zone d'activités de la Cobrelle a été décidée pour permettre l'accueil des Magasins Généraux d'Épinal qui avaient choisi de quitter leurs locaux exigus et dispersés dans Épinal pour regrouper l'ensemble de leurs activités à l'extérieur, c'est certainement la zone de Golbey 3 qui est la plus représentative d'une " zone à la carte », c'est-à-dire d'une zone profilée pour répondre de la manière la plus appropriée à la demande d'un industriel (photo 1). Cette zone créée en 1974, d'une superficie initiale de 54,2 hectares, occupée jusqu'alors par des exploitations agricoles et quelques bois, est restée vide jusqu'en 1988, date à laquelle le site golbéen a été retenu, à l'issue d'une âpre concurrence avec l'Alsace voisine, pour l'implantation de la papeterie norvégienne Norske Skog7. Cette vaste zone, menacée de fractionnement durant la longue phase d'attente d'un grand projet industriel, a été ensuite calibrée aux besoins de l'entreprise. Sa superficie a été portée à 70 hectares: pour un grand établissement industriel, l'existence d'un vaste espace non loti et que l'on fait aménager sur mesure est très intéressante. Norske Skog est le seul occupant de la zone et employait, 460 salariés, en 2003. La zone Hellieule 3, à Saint-Dié, a été aménagée en 1991 dans des circonstances analogues, c'est-à-dire pour satisfaire une demande d'entreprise, en l'occurrence, ici, MEA, société allemande spécialisée dans la fabrication de fenêtres en PVC (environ 180 salariés) ${ }^{8}$. 
Photo 1 : Norske Skog à Golbey 3 : « une zone à la carte » (2001).

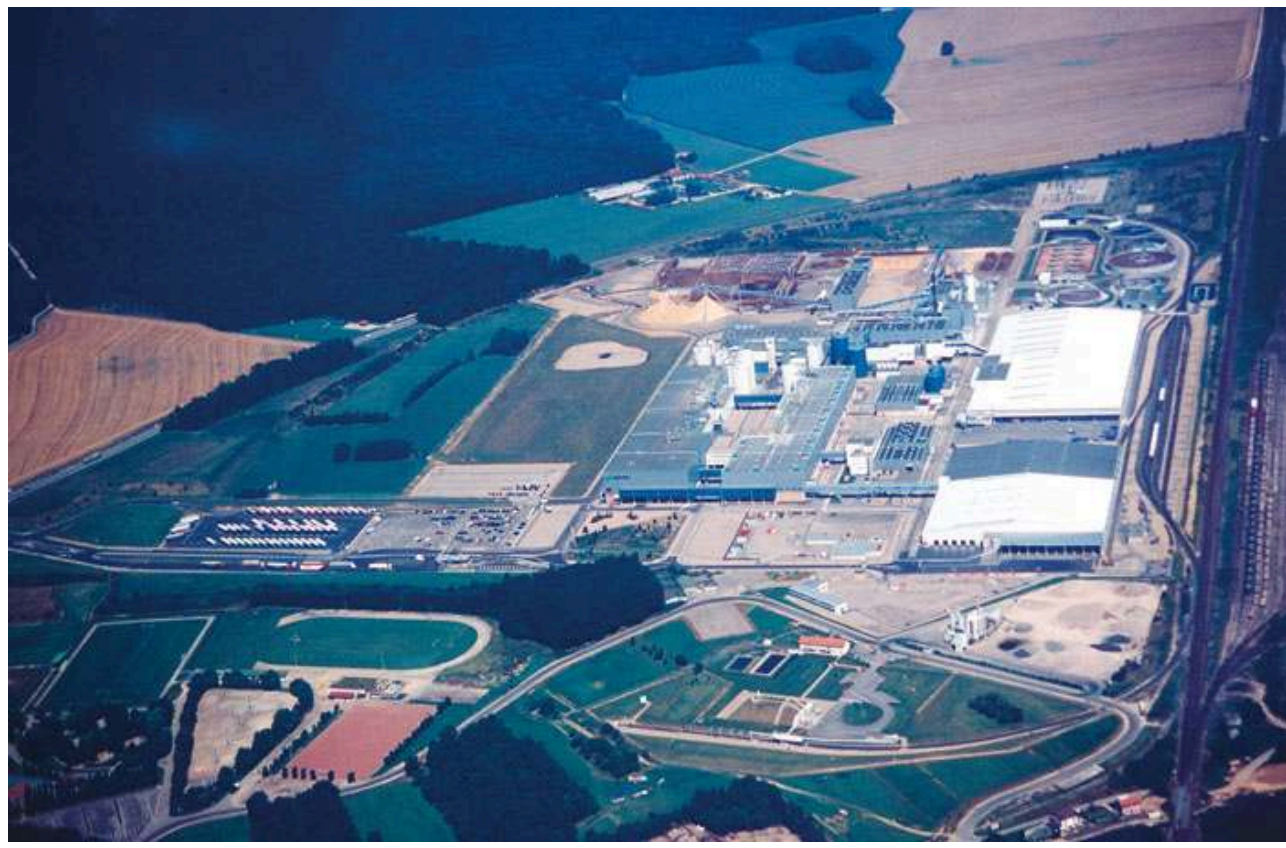

Dans un environnement apparemment très rural sur cette photographie, la zone industrielle Golbey 3 desservie par un embranchement ferroviaire de la ligne Nancy-Belfort accueille, depuis 1991, I'unique mais vaste établissement norvégien Norske Skog. Cette papeterie produit 600000 tonnes de papier journal par an à partir de bois stocké sur le parc au fond de la zone, à gauche, et de vieux papiers accumulés dans le vaste hangar blanc, en avant de la station d'épuration. Les autres bâtiments abritent d'une part les deux machines de pâte à papier, sur la gauche, d'autre part le stock de bobines de papier journal, en avant à droite. Cet établissement employait 460 salariés en 2003.

Cliché A. Humbert - C. Renard-Grandmontagne

12 Requises parfois par des entreprises dans le cadre d'une stratégie de développement de leur société, engendrées dans quelques cas par des organismes de concertation mais le plus souvent à la seule initiative d'une commune qui cherche, par l'aménagement d'une nouvelle zone, d'une part à reconvertir son activité économique, ici textile, d'autre part à augmenter ses recettes locales, les zones industrielles d'Épinal apparaissent bien peu comme une sécrétion de la ville-centre. Néanmoins, la présence de services tertiaires plus ou moins rares caractéristiques d'une ville moyenne, chef-lieu de département, ne constitue-t-elle pas un facteur attractif pour l'implantation des établissements dans les ZI de toute l'agglomération?

\section{Une occupation plus ou moins rapide des zones}

13 Dans leur ensemble, les ZI de l'agglomération spinalienne sont bien occupées, à l'exception de la zone industrielle d'Épinal-Nomexy qui, plus de 10 ans après la décision de création, ne compte que deux établissements : les Transports michel qui emploient environ 60 salariés et Innothera, entreprise pharmaceutique spécialisée dans les pathologies féminines, qui offrait 80 emplois en 1999 et emploie actuellement 145 salariés.

14 Faut-il voir dans cette difficulté de remplissage de la zone la plus éloignée l'indice de l'existence d'un seuil de distance kilométrique ou de distance-temps au-delà duquel l'attractivité serait réduite et les chances d'occupation diminuées? Avant de pouvoir éventuellement confirmer cette hypothèse peut-être faut-il examiner d'autres facteurs 
intervenant dans l'attractivité d'une zone. L'accessibilité est un facteur essentiel, très fréquemment mis en avant par les usagers. L'accessibilité de la ZI d'Épinal-Nomexy peut être appréciée comme bonne en raison de sa double desserte autoroutière et ferroviaire avec accès directs ${ }^{9}$; par sa localisation le long de la voie rapide, elle est dans une situation tout à fait identique à celle d'INOVA 3000. Le prix du foncier, en 2004, (de 16 à 18 e le $\mathrm{m}^{2}$ avant toute négociation) est comparable à celui pratiqué sur d'autres zones mais supérieur à celui de Thaon $\left(6,10 \mathrm{e} / \mathrm{m}^{2}\right.$, pour une création, $3,80 \mathrm{e}$ pour une exurbanisation). Exonérée de taxe professionnelle par la commune pour une durée de 5 ans, toute entreprise devra ensuite s'acquitter de cette taxe au taux de 9,27\% (en 2003), soit une imposition inférieure à celle de Thaon et de Jeuxey (voir tableau 2).

Tableau 2 : Taux communaux de taxe professionnelle, en 2003, pour les communes des agglomérations d'Épinal et Saint-Dié

\begin{tabular}{|l|c|l|}
\hline \multicolumn{1}{|c|}{ Communes } & Taux communal de TP en 2003 & $\begin{array}{c}\text { Part approximative de la TP } \\
\text { dans les recettes fiscales communales }\end{array}$ \\
\hline Epinal & 19,19 & non communiquée \\
\hline Golbey & 7,43 & $60 \%$ \\
\hline Jeuxey & 11,19 & $47 \%$ \\
\hline Chavelot & 5,90 & $75 \%$ \\
\hline Thaon-les-Vosges & 10,44 & $66 \%$ \\
\hline Nomexy & 9,27 & $34 \%$ \\
\hline Saint-Dié & 19,99 & non communiquée \\
\hline Saint-Michel/Meurthe & 7,08 & $68 \%$ \\
\hline Sainte-Marguerite & 6,47 & $75 \%$ \\
\hline Remomeix & 6,86 & non communiquée \\
\hline
\end{tabular}

1. La part de la TP dans l'ensemble des recettes communales est évidemment inférieure à ces valeurs ; à titre d'exemple, elle représente $18 \%$ des recettes de la commune de Jeuxey et $45 \%$ de celles de Saint-Michel-sur-Meurthe.

Source : Préfecture des Vosges pour les taux de TP ; enquête personnelle pour la part dans les recettes communales.

15 Ces différents facteurs ne semblent pas défavorables à cette zone, ce qui renforce donc l'hypothèse émise auparavant du rôle de l'éloignement de la zone. Des situations analogues peuvent être évoquées : la ZI des Sables, à Dombasle-sur-Meurthe, à environ $20 \mathrm{~km}$ de Nancy ou la ZI des Souhesmes à $11 \mathrm{~km}$ à l'ouest de Verdun sont toujours très faiblement occupées de nombreuses années après leur création.

Il semble effectivement que ces vastes zones établies en périphérie très extérieure des agglomérations éprouvent des difficultés à être occupées. Néanmoins, cette hypothèse du rôle de l'éloignement par rapport au centre de l'agglomération doit être relativisée par l'observation de la vitesse de remplissage d'autres zones plus proches du centre. Faut-il rappeler que 14 ans se sont écoulés entre la création de la zone de Golbey 3 et l'implantation de la grande papeterie. Le cas de la zone occidentale de Thaon peut être à nouveau sollicité. De 1983, date de la création, à 1998, date du changement de nom qui devient INOVA 3000, cinq établissements s'étaient installés. Depuis 1998, 35 autres établissements sont venus les rejoindre (figure 3). Cette accélération du remplissage est à mettre en partie au crédit d'une politique volontariste de l'équipe municipale élue en 1995. Cette politique comporte au moins deux volets qui visent à rendre la zone agréable pour ses usagers et désirable pour de nouvelles entreprises. Le premier volet porte sur le développement "socio-territorial de la zone ${ }^{10}$, c'est-à-dire sur l'équipement de la zone en services supplémentaires, tels «l'hôtel des services » qui 
regroupe une crèche, un centre de tri postal, un cabinet de médecine du travail, un centre de lavage des voitures, un centre de contrôle technique, un restaurant, une aire de pique-nique. De plus, en 2003, la zone INOVA 3000 s'est dotée d'un système de gardiennage. Une partie est fermée durant la nuit, l'autre étant placée sous la surveillance de rondiers. A la différence du site industriel de Ludres (54) dans lequel existe aussi, depuis 2001, un service de surveillance exercé par des rondiers auquel adhérent les entreprises volontaires, l'adhésion au service de gardiennage est obligatoire pour l'ensemble des usagers d'INOVA 3000. Quant au second volet de cette politique, c'est une importante démarche de communication qui s'exprime par la devise « Bien faire et le faire savoir » (photo 2). Cette démarche a de multiples facettes : pose de panneaux le long de la voie rapide Nancy-Remiremont, changement du toponyme de la zone qui de Thaon-ouest est devenu INOVA 3000 orthographié avec un seul $\mathrm{N}$ pour innover davantage !, importante médiatisation dans la presse locale (Est Républicain, Liberté de l'Est). La lecture de la revue de presse de la rubrique «zones d'activités » effectuée par la CCI d'Épinal témoigne de l'abondance des articles relatifs à cette zone et au foisonnement d'idées de son maire.

Figure 3 : Plan de la zone industrielle INOVA 3000 (Thaon-les-Vosges).

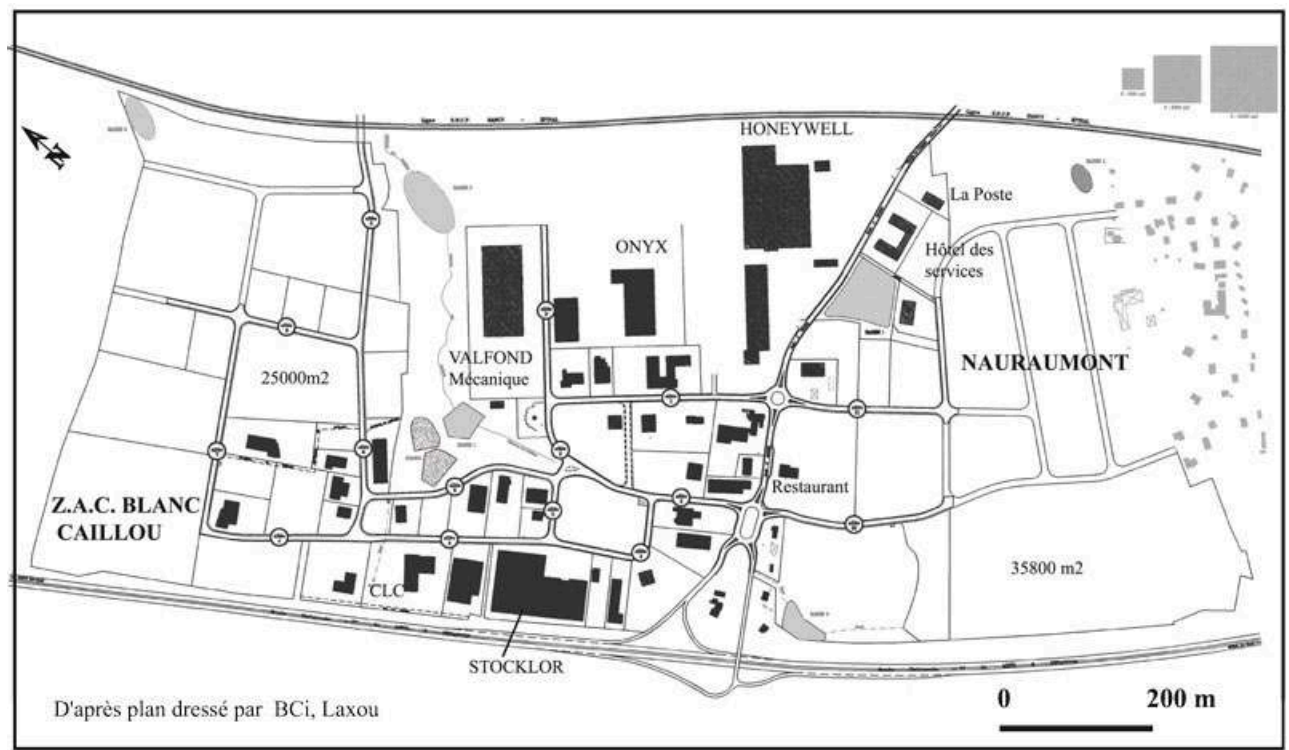

Ce plan d'INOVA 3000 indique l'occupation de la zone et son extension en 2003. L'établissement le plus ancien est celui de Garrett-Honeywell (1979) situé près de la voie ferrée. L'embranchement ferroviaire qui le desservait n'est plus fonctionnel et a même disparu du plan. Seuls la voie routière et son accès direct sont utilisés. Cette voie rapide est la plus fréquentée du département avec un flux de 30000 véhicules par jour.

Alors que la partie centrale est presque totalement occupée, la troisième extension de la zone portant sa superficie à 120 hectares s'effectue simultanément au sud (projet d'implantation d'un hôtel) et au nord, en limite de la commune de Thaon. Le réseau viaire est déjà prolongé. De la même façon que le nom de la zone a été modifié, celui des rues l'a été également : la rue de l'Avenir est devenue Allée 1 ; une odonymie de type américain a été préférée. La zone INOVA 3000 est devenue la plus importante en superficie du département. 


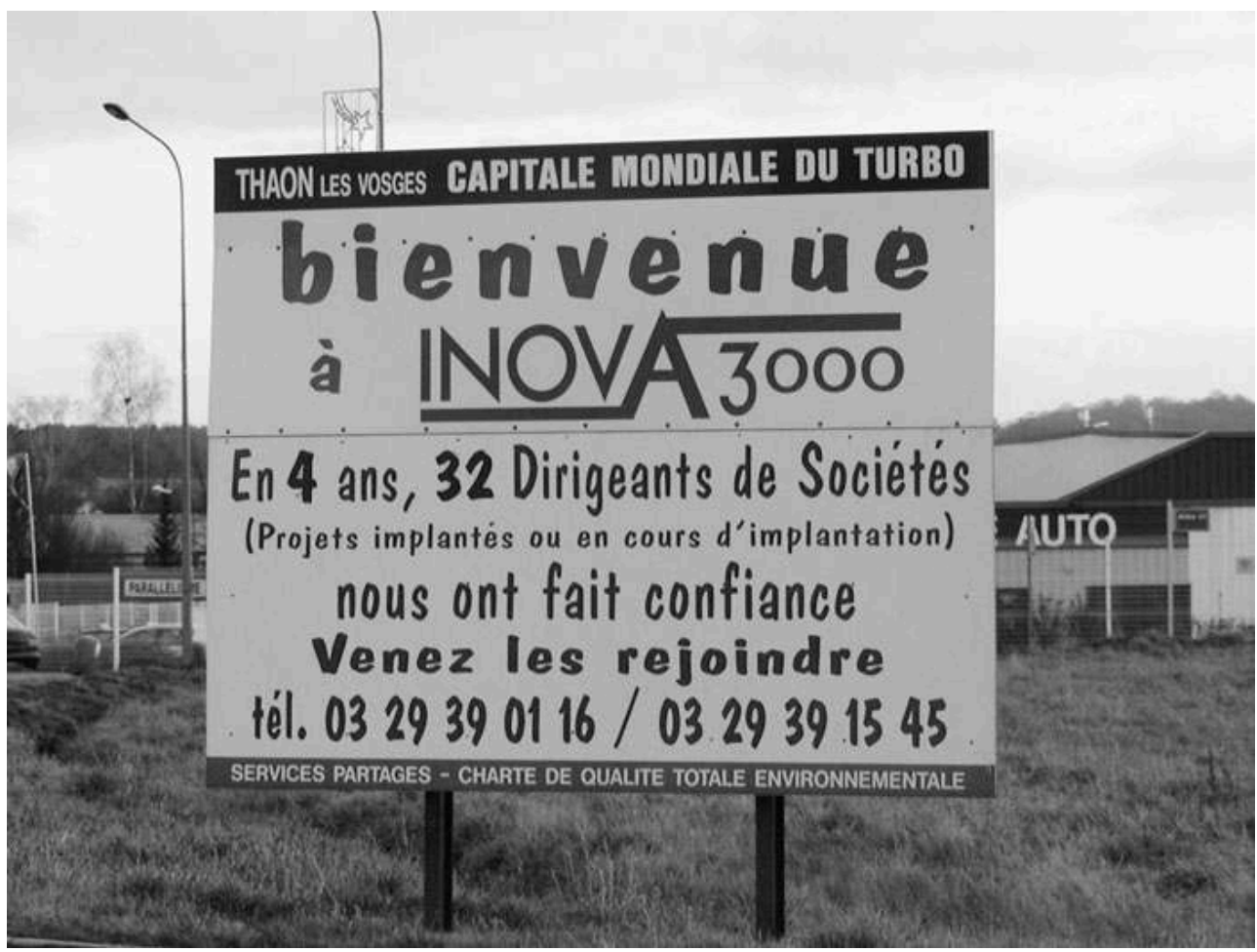

Ce panneau n'est pas le panneau standardisé situé le long de la voie rapide. II se dresse dans la zone INOVA 3000, à l'entrée, en face du mobilier urbain qui porte le plan de la zone. II rappelle que

l'établissement américain Garrett- Honeywell fut le premier à s'implanter. C'est le principal employeur de la zone et confère le titre de « capitale mondiale du turbo-compresseur » à Thaon. Ce panneau rend compte de deux des trois qualités que la municipalité veut voir reconnues à la zone : la qualité environnementale par la signature d'une charte (cependant la zone n'est pas certifiée), le dynamisme par l'accélération de son occupation depuis 1998.

Cliché C. Renard-Grandmontagne

17 A la lumière d'autres études de développement de zones industrielles en Lorraine et en France, il semble bien que l'énergie déployée par une équipe municipale en faveur de sa ou ses zones et que l'importance consacrée à la politique de communication sur ce sujet soient des éléments déterminants d'occupation des zones. Il n'est pas sûr qu'une structure supra-communale puisse s'investir de la même manière.

En ce début de XXI ${ }^{e}$ siècle, il n'existe pas encore de communauté d'agglomération à Épinal; des contacts et des négociations sont en cours. Deux communautés pourraient naître : une communauté d'agglomération centrée sur Épinal et une communauté de communes qui regrouperait les communes du canton de Châtel-sur- Moselle grossie d'une partie de celles du canton de Charmes. La mise en place de telles communautés modifiera probablement les relations entre collectivités et zones.

\section{Saint-Dié : un développement récent et surtout communal des zones d'activités}

19 En quoi les zones d'activités de l'agglomération de Saint-Dié se différencient-elles de celles d'Épinal ? Sont-elles davantage des sécrétions de la ville centre? Un regard, même rapide, sur les deux cartes de la figure 4 révèle immédiatement une première différence entre les deux villes-centres. Alors qu'une seule ZI a été aménagée sur le 
territoire de la ville d'Épinal, ce sont quatre voire cinq zones qui le sont à Saint-Dié, principalement le long de la Meurthe, en aval de la ville. La commune a pu disposer là de terres agricoles souvent humides car situées en zone inondable. Le toponyme «Hellieule ", attribué à toutes les zones, coïncide d'ailleurs avec l'emplacement des anciennes prairies de fond de vallée et l'aménagement des ZI a nécessité la rectification du cours de la Meurthe (Photo 3).

Figure 4 :a. Les zones d'activités de l'agglomération d'Epinal. b. Les zones d'activités de l'agglomération de Saint-Dié.

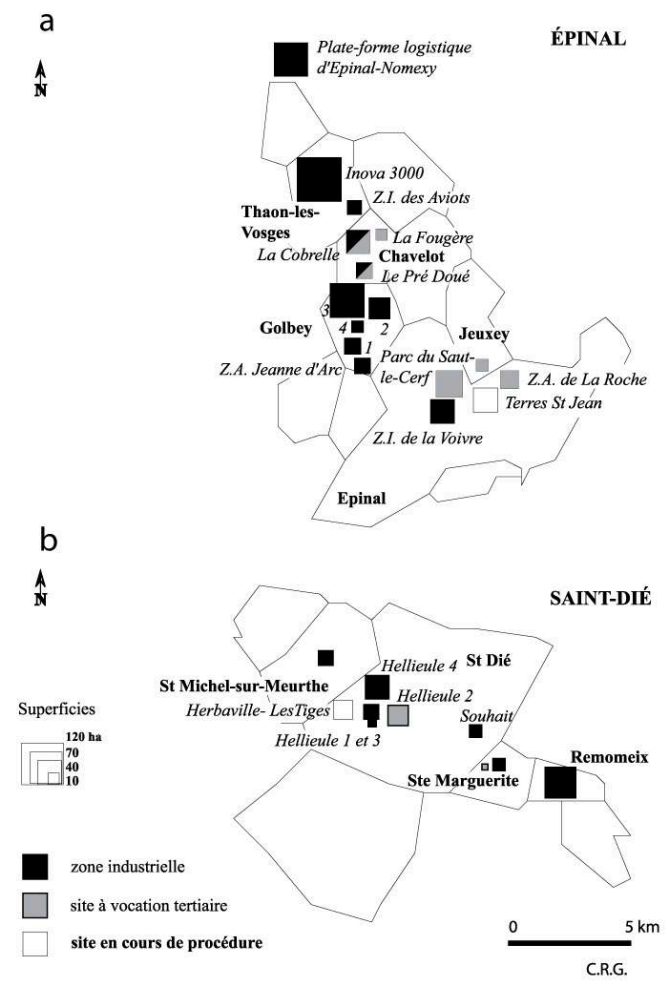


Photo 3 : Zones d'activités déodatiennes (2002)

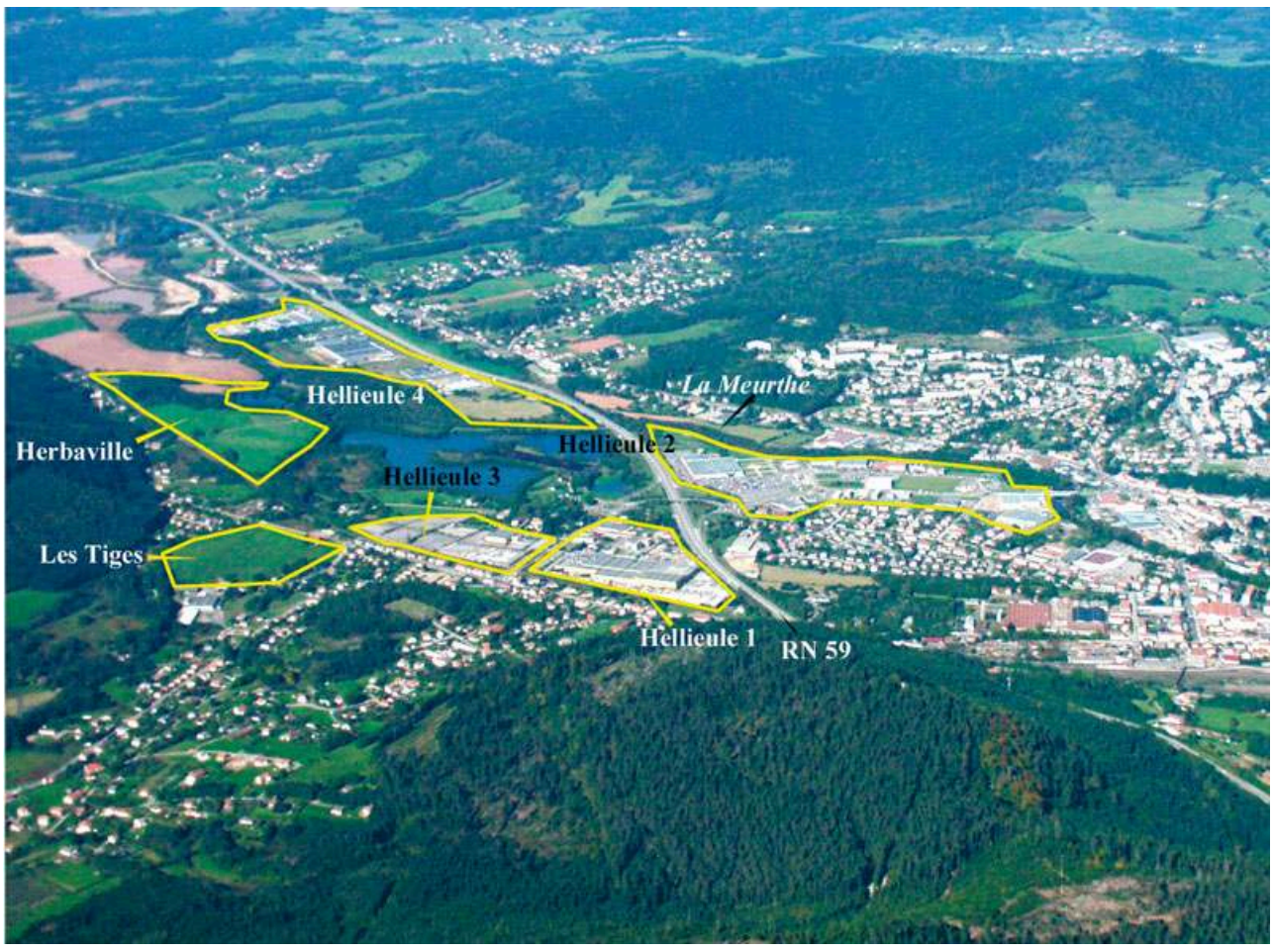

La périphérie nord de la ville de Saint-Dié s'avance jusqu'au milieu du cliché. Dans la vallée de la Meurthe dont le cours a été rectifié, ont été aménagées quatre des cinq zones d'activités de la ville. Ce sont les zones Hellieule créées au début des années 1990 à l'emplacement d'anciennes prairies inondables. Chacune d'entre elles a reçu une fonction spécifique : industrielle, commerciale ou logistique. Elles sont toutes directement desservies par la RN 59

Cliché A. Humbert - C. Renard-Grandmontagne

20 La seconde différence apparaît aussi nettement sur les cartes de la figure 2. Celle-ci nous renseigne sur la période de création de ces zones. A l'exception d'Hellieule 1 qui est un regroupement spatial spontané de quelques établissements artisanaux et de l'établissement industriel MEA installé en septembre 1989, toutes les autres zones de Saint-Dié ont été créées simultanément en 1991, soit peu de temps après l'ouverture de la voie rapide de contournement de Saint-Dié, fin 1989. Hellieule 2, Hellieule 3 et Souhait ont d'ailleurs fait l'objet de la même procédure de création, celle d'un parc d'activités multi-sites. La SEV (Société d'Équipement Vosgienne) est l'aménageur de ces zones déodatiennes. C'est aussi une société d'économie mixte d'aménagement et de gestion créée en 1990, dont le capital est constitué majoritairement par des fonds publics de la ville de Saint-Dié $(61,24 \%)$ mais aussi de Neufchâteau, Moyenmoutier, Raon-l'Étape, Fraize, Bruyères. Si Hellieule 2 et Hellieule 3 sont des zones créées ex nihilo, celle de Souhait offre l'exemple d'une création de zone à partir d'un établissement préexistant: Arvin M eritor (ex Rockwell), équipementier automobile d'origine américaine, implanté à Saint-Dié en 1960 dans une ancienne usine textile et qui emploie environ 1000 salariés. Cette petite zone intra-urbaine a une superficie totale de 9 hectares dont seulement 3,5 ont fait l'objet d'une procédure de ZAC en 1991. Il existe, en Lorraine, d'autres exemples similaires de bourgeonnement de zones industrielles à partir d'un "singleton »: Eurotransit, au nord de Metz, créé, dans les années 1980 et 90, autour de la SMAE (Société Mécanique des Automobiles de l'Est, groupe PSA) construite en 1979. La ZI de Baleycourt, à Verdun, a été créée en 1975 dans 
le prolongement de deux implantations industrielles antérieures : la firme britannique de chimie organique ICI-France, déjà installée en 1965, et l'usine de traitement des résidus laitiers Lacto-Serum France, créée en $1966^{11}$. l'agglomération ( $56 \mathrm{ha}$ ), à Remomeix. Mais cette zone n'est pas une sécrétion déodatienne; il s'agit d'une zone dite d'intérêt départemental décidée par le Conseil Général des Vosges, en 1996. L'observation de sa gestation ne manque pas d'intérêt. En effet, en 1995, le Conseil Régional a proposé à chacun des quatre départements lorrains de financer l'aménagement d'une vaste ZI (environ $100 \mathrm{ha}$ ), vaste plate-forme d'accueil capable de recevoir des projets industriels d'envergure. Le Conseil Général des Vosges, officiellement plus soucieux d'équilibrer ses territoires, a préféré, dans un premier temps, fractionner le projet entre ses quatre bassins d'emploi, puis finalement les attribuer à deux seulement, celui de Neufchâteau avec le projet de Châtenois et celui de Saint-Dié avec la zone de Remomeix. Des concurrences et des affrontements entre conseillers généraux ont abouti, le 2 décembre 1996, à l'adoption de deux décisions plus complexes, l'une associant la zone franche de Mirecourt-Juvaincourt à celle de Châtenois, l'autre créant une zone bipolaire dite de Remomeix-Lusse, en milieu rural, à proximité de la RN 59. Parallèlement à la procédure traditionnelle de création d'une ZAC, une procédure P.A.L.M.E (Parc d'Activités Labellisé pour la Maîtrise de l'Environnement) a été lancée. La future zone devra être conforme aux normes environnementales «ISO 14001 » en matière de prise en compte des caractéristiques du site, de gestion de l'eau, des déchets et de l'air, et de la qualité de vie des riverains. Sept ans plus tard, l'enquête préalable à la déclaration d'utilité publique (DUP) est terminée, ainsi que l'enquête désormais exigée par la loi sur l'eau. La DUP devrait être obtenue au début de l'année 2004, permettant ensuite l'acquisition de terrains.

Enfin, une troisième différence apparaît entre les zones déodatiennes et spinaliennes. Si les zones spinaliennes sont identifiées par l'implantation d'un grand établissement («Golbey 1, c'est la Trane », «Golbey 2, c'est Michelin»), les zones de Saint-Dié sont caractérisées par la fonction qui leur a été attribuée par la ville. Si Hellieule 1, Hellieule 3 et Souhait ont une vocation purement industrielle, Hellieule 4 est réservée à la logistique et aux transports. Les deux principaux établissements sont d'une part solotra-Hermann (250 salariés en 2001) qui assure principalement la logistique et le transport pour le compte d'équipementiers automobiles de l'Est de la France vers l'Allemagne, la Suisse, l'Italie et l'Europe de l'Est, d'autre part la base de stockage de produits frais d'Intermarché (280 salariés). Hellieule 2 est consacrée principalement au commerce et aux services. Quant à la future zone d'Herbaville-Les Tiges, elle sera partagée entre une aire d'accueil pour des artisans et des industriels et un espace de loisirs de plein air avec la réalisation d'un " géoparc » à proximité des étangs.

La spécialisation des zones est donc nettement plus accusée à Saint-Dié qu'à Épinal. Néanmoins, l'étude détaillée du contenu de ces zones révèle la présence de deux imprimeries, l'une dans la zone commerciale d'Hellieule 2 et l'autre dans la zone d'Hellieule 4. Ces quelques entorses tempèrent à peine la mono-fonctionnalité délibérée. 


\section{centre, plus specalses en rason diune governance pinciple, les zones dact centre, plus spécialisées en raison d'une gouvernance principale, les zones d'activités de l'agglomération de Saint-Dié semblent davantage des sécrétions de la ville, à l'exception de celle de Remomeix. \\ III. Le développement récent et rapide des zones commerciales périphériques}

Beaucoup moins nombreuses, les zones d'activités commerciales, par ailleurs plus petites (de 8 à $43 \mathrm{ha}$ ), ne représentent qu'environ le cinquième de l'ensemble des zones d'activités dans chacune des deux agglomérations. Comme dans de nombreuses autres villes, elles sont plus proches du centre de l'agglomération que les zones industrielles et se partagent, ici, entre banlieue proche et lisière de la ville-centre (figure 4).

Deux grands types de concentrations de commerces ${ }^{12}$ sont identifiables dans chacune des deux agglomérations. Ce sont tout d'abord les concentrations de commerces entraînées par une locomotive incontestée, c'est-à-dire une grande surface généraliste. Cette locomotive, toujours un hypermarché, a été en général le précurseur de la zone. Ainsi, les Coopérateurs de Lorraine ont ouvert, à Jeuxey, en 1983, une grande surface qui, depuis, a changé deux fois d'enseigne (Euromarché puis Carrefour). Longtemps isolé, cet hypermarché est maintenant entouré d'une demi-douzaine d'enseignes nationales ou internationales telles que Jouet Club, Gemo, Intersport, Mc Donald's, Darty.

En amont de Saint-Dié, à Sainte-Marguerite, l'hypermarché Cora a ouvert ses portes en 1973. Se sont agglutinés, à proximité (dans la zone d'activités des Pierres du Faing) et le long de la RN $420^{13}$, de nombreux commerces qui constituent, en quelque sorte, une zone corridor ${ }^{14}$. Cette concentration de commerces fut longtemps la seule de l'aire de chalandise de Saint-Dié.

Depuis la fin des années 1990, l'ouest déodatien est rééquilibré par l'aménagement de Hellieule 2 sur laquelle a été transféré le centre Leclerc (photo 4). Cette zone a fait l'objet d'une annexe particulière au règlement d'aménagement de zone. Des prescriptions architecturales particulières doivent «principalement viser à magnifier une nouvelle entrée de la Ville, à concevoir des espaces de convivialité adaptés à chaque produit, à s'intégrer à l'environnement paysager du site et à se greffer sur les quartiers périphériques d'habitation $»^{15}$. A la prescription commune à l'ensemble des zones Hellieule 2, Hellieule 3, Souhait, limitant l'emprise au sol des constructions à $50 \%$ de la superficie des parcelles, s'ajoutent d'autres prescriptions interdisant les clôtures et prévoyant l'obligation, pour les propriétaires de parcelles, de planter des arbres tiges de type Acer platanoïdes d'un diamètre minimum de 16 à $18 \mathrm{~cm}$ et d'une hauteur de 2,50 $m$ sous branches, afin de créer un espace ouvert et rapidement végétalisé. Quant aux bâtiments qui doivent être alignés, ils peuvent être pourvus de bardages métalliques, à condition qu'ils soient de couleur gris clair, comme les autres constructions ; mais les façades le long des rues doivent être largement vitrées, c'est-à-dire sur au moins le tiers de leur longueur. Seules les enseignes placées sur les bâtiments sont autorisées mais la signalisation des activités peut être aussi assurée par un mât support de drapeaux. Ces mâts sont fournis et posés par l'aménageur. Toutes ces dispositions visent, bien entendu, à la recherche d'homogénéité sinon d'harmonie. Ces contraintes n'ont pas 
découragé les candidats à l'installation : cette zone s'est remplie presque totalement depuis 2001.

Photo 4 : La zone d'activités Hellieule 2, à Saint-Dié (2002)

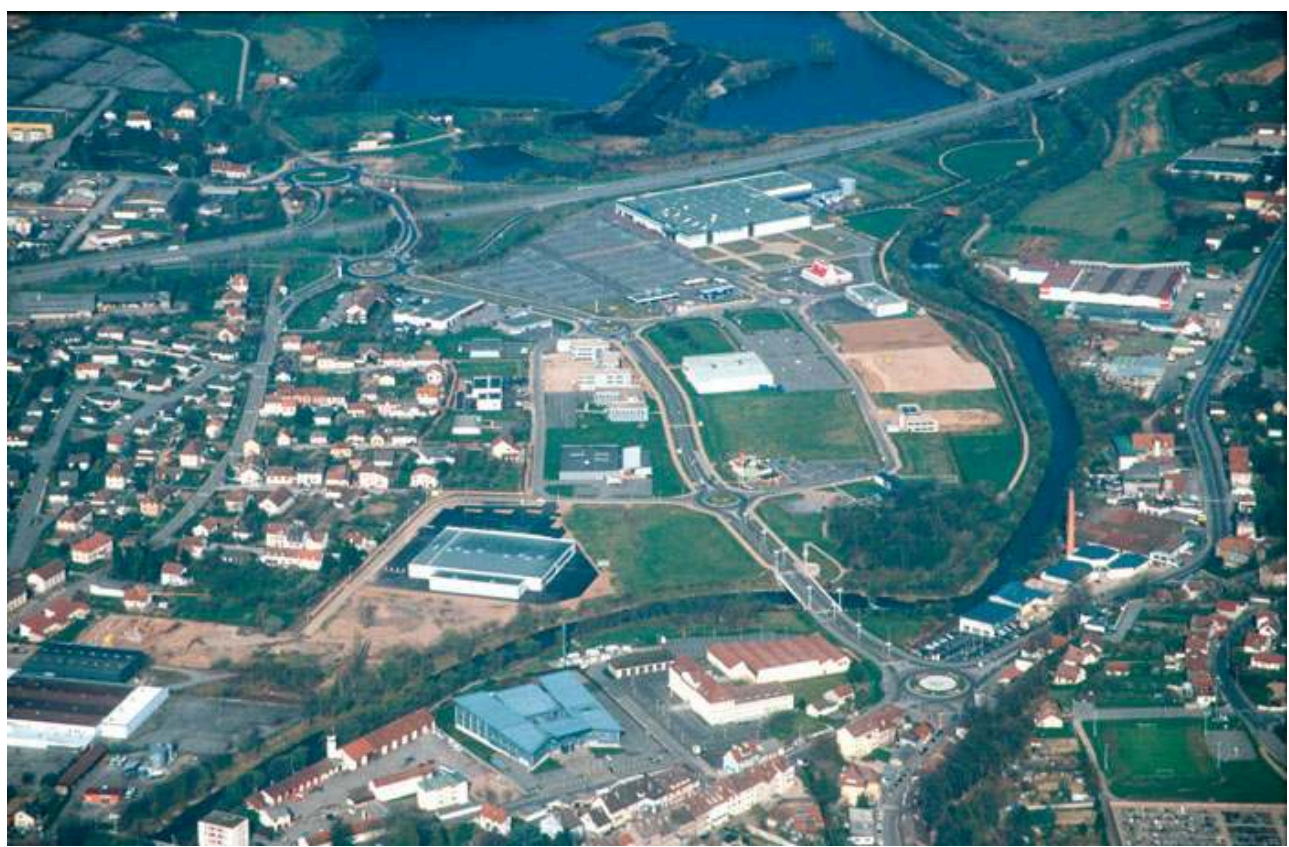

Entre Meurthe et rocade de contournement de Saint-Dié, ce plan rapproché de la zone Hellieule 2 met en évidence le contact entre espaces résidentiels et espaces d'activités. Les contrastes architecturaux sont évidents entre les anciennes usines aux toits de shed, sur la rive droite de la Meurthe, le bâtiment moderne de l'IUT visible au premier plan et les formes parallélépipédiques des nouveaux établissements de la zone à vocation surtout commerciale. Depuis la prise de vue de cette photographie, en 2002, cette zone s'est encore remplie. Elle compte 37 établissements et constitue un des deux pôles commerciaux périphériques situés respectivement à l'amont (Sainte-Marguerite) et à l'aval de l'agglomération

Cliché A. Humbert - C. Renard-Grandmontagne

D'autres structures de concentrations de commerces existent: ce sont les concentrations dans lesquelles les commerces ont globalement la même taille (petite ou grande), sont spécialisés ou non ${ }^{16}$. Ce sont, dans l'agglomération d'Épinal, la zone commerciale de la Fougère, le parc d'activités de la Roche, le lotissement du Pré Droué et le parc économique du Saut-le-Cerf. La zone commerciale de la Fougère, d'initiative privée, est la plus ancienne de toutes les zones commerciales d'Épinal, mais aussi, vraisemblablement, de tout le département des Vosges. Elle jouxte la voie rapide Nancy-Remiremont vers laquelle elle dispose d'un accès direct. Elle compte 46 établissements principalement disposés le long de deux rues convergentes. Sans qualité esthétique, vieillissante, cette zone est néanmoins encore très fréquentée.

31 Sur la même commune de Chavelot, dû également à un promoteur privé, le lotissement industriel et commercial du Pré Droué, de type corridor, regroupe principalement des commerces liés à l'automobile et à la moto. Les concessionnaires automobiles et de motos figurent, avec les exposants de meubles, parmi les commerces qui, les premiers, se sont regroupés spatialement. On retrouve une telle grégarité de commerces spécialisés dans une branche - en particulier l'automobile - dans de nombreuses agglomérations : c'est le "village de l'auto ", dans la zone d'activités économiques de 
Capnord, au nord-est de Dijon ou le «Polygône" à Laxou pour l'agglomération nancéienne.

Enfin, les deux zones commerciales les plus récentes furent aménagées sur la commune d'Épinal, par la collectivité elle-même, sur deux pénétrantes routières. Ce sont les plus vastes: le Parc Économique du Saut-le-Cerf s'étend sur 43 hectares et la Zone d'Activités de la Roche occupe 20 hectares (photo 5). Certes, elles ne sont pas exclusivement commerciales, mais l'activité commerciale y est prédominante et elle est à l'origine de congestions redoutables des voies d'accès, en particulier en fin de semaine. Pourquoi ces nouvelles zones sont-elles nées alors que, rappelons-le, elles n'hébergent pas d'hypermarché ? C'est que la concurrence est plus lointaine. En effet, en 1992, est enfin ouverte l'intégralité de la voie rapide Nancy-Épinal qui met la préfecture vosgienne à moins de $45 \mathrm{mn}$ de la cité ducale et de son offre commerciale. Cette réduction de la distance-temps entre ces deux agglomérations laissait craindre, pour les commerçants d'Épinal, un accroissement de l'évasion commerciale vers Nancy. Il s'est agi, pour la collectivité spinalienne, de renforcer l'offre commerciale de la ville en créant de nouvelles zones pour accueillir des unités de vente dans les créneaux les moins bien dotés, à savoir les secteurs sports et loisirs ainsi que le bricolage ${ }^{17}$. La création du Parc économique du Saut-le-Cerf répond à un certain nombre d'objectifs : « renforcer la zone de chalandise d'Épinal, en empêcher toute évasion de clientèle, sans provoquer de transferts de clientèle du centre-ville vers la zone, la priorité pour la commercialisation étant donnée aux investisseurs du cru ${ }^{18}$. 
Photo 5 : Zones d'activités spinaliennes (2001)

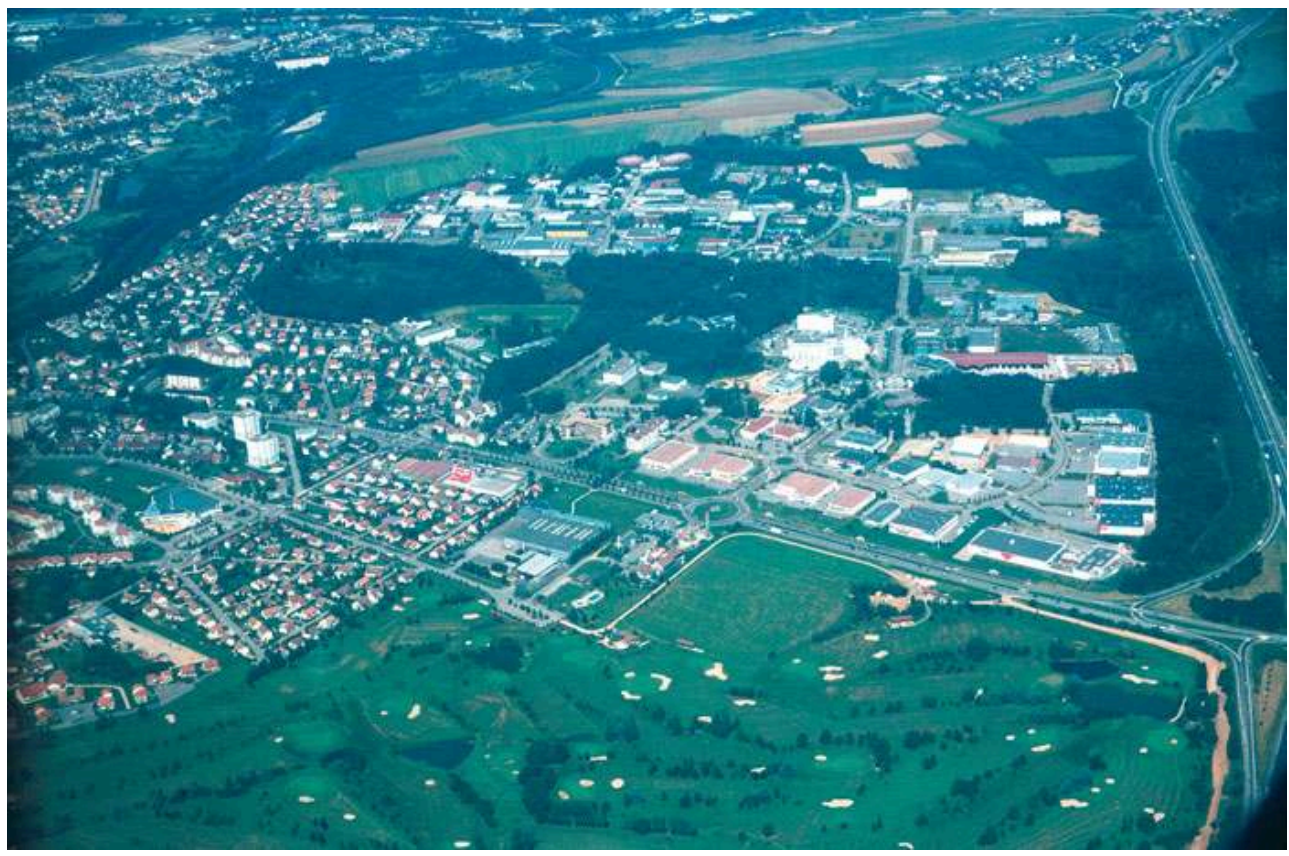

Ce cliché présente dans sa totalité les deux principales zones d'activités de la ville d'Épinal. Au fond au contact du terroir agricole, la zone industrielle de la Voivre (35 ha) a été créée en 1974, à la limite septentrionale de la commune. En avant du bois de la Voivre, le Parc économique du Saut-le-Cerf (43 ha), créé en 1988, regroupe des services tertiaires (URSSAF, CAF, Clinique, Archives départementales) et des enseignes commerciales appartenant, le plus souvent, à des groupes nationaux (Fly, Thiriet, Hygena, par exemple), très visibles depuis la pénétrante qui se greffe sur la voie rapide repérable à l'extrémité droite de la photographie. D'autres commerces ont profité de cette concentration pour s'installer mais à l'extérieur du parc : Jardiland et Buffalo Grill, ce dernier aisément reconnaissable à son toit sang de bœuf. Au contact de ces zones, d'autres espaces fonctionnels ont été aménagés : les lotissements pavillonnaires du Saut-le-Cerf, I'ENSTIB (École nationale Supérieure des Techniques et des Industries du Bois) et le vaste golf d'Épinal (40 ha).

Cliché A. Humbert - C. Renard-Grandmontagne

33 L'initiative de la ville ne se limite pas à la création d'une zone commerciale accompagnée ou non d'un cahier des charges plus ou moins contraignant; elle peut aussi intervenir en orientant le contenu de la zone. L'occupation d'une zone commerciale résulte d'une subtile alchimie qui mêle la stratégie d'implantation d'une société (Buffalo Grill, par exemple) ou d'un groupe (Accor ou Envergure) à la volonté municipale de provoquer les implantations de certaines enseignes, mais aussi de sélectionner les candidats à l'ouverture par sa participation influente à la $\mathrm{CDEC}^{19}$. C'est ainsi que les édiles spinaliens sont intervenus, avec succès, auprès de Decathlon pour obtenir une ouverture, fin 1999, en face de la zone d'activités de la Roche qui a d'ailleurs été stimulée par cette implantation. Une démarche tout à fait similaire a été conduite par les élus déodatiens auprès des sociétés Decathlon et Bois et Chiffons ${ }^{20}$. Certaines enseignes nationales et même internationales apparaissent, au moins momentanément, suffisamment prestigieuses pour que leur implantation draine une clientèle supplémentaire dans une aire de chalandise élargie. D'autre part, l'installation de ces enseignes nationales, que leurs magasins soient franchisés ou qu'ils ne le soient pas, semble accroître la notoriété d'une ville ou de son agglomération, et par la même la satisfaction de ses élus. Inversement, semble exister un sentiment de frustration quand ne s'illumine pas une enseigne convoitée. L'enseigne lumineuse verte de LeroyMerlin en est une et l'ouverture d'une de ses grandes surfaces spécialisées, début 2004, 
à la sortie sud de l'agglomération nancéienne inquiète les responsables en charge du dossier du commerce à Épinal.

La politique de quadrillage systématique du territoire national par de grands groupes nationaux ou internationaux, la concurrence entre les agglomérations distantes de plusieurs dizaines de kilomètres (de 50 à $100 \mathrm{~km}$, environ), mais aussi la motorisation de consommateurs plus disponibles et de plus en plus attirés par les grandes enseignes mais n'hésitant pas à pratiquer le "zapping commercial », expliquent la banalisation du contenu de ces zones commerciales périphériques.

Cette étude a dévoilé la diversité d'une des manifestations fréquentes et banales de la croissance urbaine et de la périurbanisation. Elle a souligné la différenciation très nette entre zones industrielles et zones commerciales. Alors que toutes les zones industrielles étudiées sont conçues et aménagées par les collectivités, les zones commerciales peuvent être d'origine privée. Plus proches des concentrations de population, plus récentes, les zones commerciales sont occupées plus rapidement et plus complètement que les zones industrielles et cela malgré le prix du foncier beaucoup plus élevé : à Saint-Dié le rapport est de 1 à 4 . Mais surtout, tout au long de cet article, nous avons souligné le rôle indéniable des collectivités dans la conception, l'aménagement et l'occupation de ces zones. L'occupation des zones d'activités, en particulier celles à vocation industrielle, peut être favorisée aussi par l'intervention du $\mathrm{CAPEV}^{21}$. Néanmoins, le rôle de la ville-centre d'une agglomération est modulé, d'une part par des spécificités locales telles que la structure de l'agglomération, son passé industriel, sa composition politique qui introduisent des concurrences à l'intérieur de l'agglomération et, d'autre part, par des initiatives d'acteurs économiques qui mettent en concurrence d'autres territoires.

Les deux agglomérations vosgiennes sont deux bons exemples de la complexité de ces mécanismes. Alors que l'agglomération de Saint-Dié est un type d'agglomération moyenne où la ville-centre joue un rôle essentiel, peut-être en raison du caractère très rural de sa banlieue, l'agglomération spinalienne est davantage représentative d'une agglomération polynucléaire dans laquelle chaque noyau urbain a développé, peut-être aussi en raison de son passé industriel textile, sa propre politique d'aménagement des zones, et dans laquelle il existe des initiatives privées, notamment en matière de zones commerciales. Les dispositions récentes adoptées en faveur de l'intercommunalité atténueront probablement les concurrences internes. Elles n'affecteront vraisemblablement pas les stratégies privées et renforceront peut-être les concurrences entre agglomérations.

\section{BIBLIOGRAPHIE}

BERTRAND Sabine (1979). - Les zones industrielles de Golbey : leurs implications humaines et urbaines, Mémoire de maîtrise, Nancy, Université Nancy 2. 
DESSE René-Paul (2001). - Le nouveau commerce urbain. Dynamiques spatiales et stratégie des acteurs, Rennes, Presses Universitaires de Rennes, Col. Espaces et territoires, 198 p.

EDELBLUTTE Simon (1997). - Paysages et organisation de l'espace en Lorraine. La vallée de la Moselle d'Épinal à Neuves-Maisons, Thèse de doctorat, Université Nancy 2, 498 p.

EDELBLUTTE Simon (1999). — Les difficultés imprévues d'une grosse implantation industrielle étrangère : le cas de la papeterie norvégienne Norske Skog à Golbey (Vosges), in GOUESET V. (dir), Investissement étranger et milieu local, Rennes, Presses Universitaires de Rennes, p. 373-384.

GASNIER Arnaud (2002). - Multiplexes et parcs de loisirs périurbains : de nouvelles centralités ?, Lieux de culture, culture des lieux, (ouvrage collectif), Presses Universitaires de Rennes.

LEBRUN Nicolas (2002). - Centralités urbaines et concentrations de commerces, Université de Reims, Thèse de doctorat, $490 \mathrm{p} .+$ annexes.

VAIVRE Barbara (2001). - Thaon-les-Vosges. Impact actuel et passé de l'industrie sur les paysages de Thaon-les-Vosges, Université Nancy 2, Mémoire de maîtrise en géographie, 70 p.

\section{NOTES}

1. Définition de l'INSEE.

2. L'expression « zone industrielle » a d'abord été la plus utilisée avant de céder progressivement la place à l'appellation « zone d'activités ». Les ZI apparaissent à partir de 1946 dans les plans de reconstruction des villes sinistrées afin de regrouper de manière rationnelle les activités. En fait, il a fallu attendre la loi du 14 août 1954 puis celle du 2 avril 1955 sur la décentralisation pour assister aux premières tentatives de développement organisé des zones industrielles en France. Peu à peu l'expression ZI s'est révélée trop restrictive au regard des activités plus diversifiées qui s'implantaient dans ces espaces spécifiques. Néanmoins, il n'y a pas remplacement complet de la première dénomination par la seconde, ni stabilisation des expressions. Quant à la terminologie «zone commerciale ", elle accompagne l'émergence de la grande distribution à la périphérie des agglomérations. Faut-il rappeler la naissance du premier hypermarché français à Sainte Geneviève-des-Bois, en région parisienne, en 1963? Les premières zones périphériques d'activités commerciales datent des années 1960 et 1970 mais elles sont encore rares (Plan de Campagne, près de Marseille, en 1967, Englos, à l'ouest de Lille, en 1969 et Dijon Quétigny en 1976). Elles se sont surtout développées depuis le milieu des années 1980, c'est-à-dire depuis moins de 20 ans.

3. La ZI des Aviots (ex ZI Sud à Thaon-lès-Vosges) est, en fait, l'ancien site de la BTT (Blanchisserie et Teinturerie de Thaon) en voie de reconversion et rebaptisé ZI ; c'est pourquoi il apparaît comme étant antérieur à 1970 .

4. S. Bertrand, Les zones industrielles de Golbey. Leurs implications humaines et urbaines, Mémoire de Maîtrise, Université Nancy 2, 1979.

5. Article de presse : La plus grosse zone industrielle du département est née, La Liberté de l'Est, 4 juillet 1992.

6. D'après l'annuaire 2003 des SEM édité par la fédération des SEM, le capital de la SEBL, d'origine à la fois public et privé, était de $950000 €$; il est détenu, très majoritairement par les collectivités régionales : Région Lorraine, les quatre départements ainsi que par des organismes privés : Caisse de Dépôts et de Consignations, Houillères du Bassin de Lorraine, Société ARBED, Chambre Régionale de Commerce et d'Industrie de Lorraine, Chambre Régionale d'Agriculture, CCI de Meurthe-et-Moselle, CCI de Moselle, CCI d'Épinal, CCI de Saint-Dié, Crédit Local de FranceDexia. 
7. Voir Simon Edelblutte, Paysages et organisation de l'espace en Lorraine. La vallée de la Moselle d'Épinal à Neuves-Maisons, Thèse de doctorat, Université Nancy 2, 1997, ainsi que, Les difficultés imprévues d'une grosse implantation industrielle étrangère : le cas de la papeterie norvégienne Norske Skog à Golbey (Vosges), in Goueset V. (dir), Investissement étranger et milieu local, Rennes, Presses Universitaires de Rennes, 1999, p. 373-384.

8. D'autres cas similaires existent en Lorraine. Ils ne sont pas toujours d'origine industrielle; ils sont parfois tertiaires. C'est ainsi que la grande surface spécialisée Ikeaet sa plate-forme logistique occupent la zone de la Maxe, au nord de Metz, spécialement aménagée à sa demande.

9. Elle est longée par la voie ferrée et par le canal de l'Est branche sud, mais au long de cette voie, il n'existe pas de port.

10. Expression de R. Degemard, maire de Thaon-lès-Vosges, Vice-Président du Conseil Général des Vosges.

11. Archives Départementales de la Meuse, 1699 W 9.

12. Expression introduite par Nicolas Lebrun, dans sa thèse Centralités urbaines et concentrations de commerces, Université de Reims, 2002. Pour l'auteur, une concentration de commerces recouvre toutes les différentes formes d'agrégats marchands dont les centres commerciaux, les galeries marchandes, les lotissements commerciaux mais aussi des agglomérats spontanés de magasins.

13. "Le long de la RN 420, c'est une succession de commerces, d'activités de services et autres halls d'exposition ...accolés de manière quelque peu anarchique sans beaucoup de cohérence au gré des ventes des terrains agricoles !» (Extrait d'un article de l'Est Républicain titré "SainteMarguerite en pleine expansion » du 10 novembre 1993).

14. Expression utilisée par Arnaud Gasnier dans Multiplexes et parcs de loisirs périurbains : de nouvelles centralités ?, in M. Gravari- Barbas et Ph. violier (dir), Lieux de culture, culture des lieux, Rennes, Presses Universitaires de Rennes, Col. Espaces et territoires, 2003.

15. SEV - Parc d'activités d'Hellieule 2, Annexe au plan d'aménagement de zone, janvier 1999.

16. Nicolas Lebrun les qualifie de concentrations de commerces de type « astéroïde ».

17. Selon les résultats d'une étude menée par la CCI d'Épinal en 1997, à la demande de la municipalité spinalienne.

18. Philippe seguin, député-maire d'Épinal, Liberté de l'Est, 15 mars 1989.

19. CDEC : Commission départementale d'équipement commercial, présidée par le préfet qui ne prend pas part au vote ; elle est composée de 6 membres : 3 élus locaux (le maire de la commune d'implantation, un représentant de l'établissement public de coopération intercommunale, le maire représentant la commune la plus peuplée de l'arrondissement autre que la commune d'implantation), 2 représentants des activités commerciales et artisanales (le président de la CCI et le président de la Chambre des Métiers concernés par le projet) et 1 représentant des associations de consommateurs du département.

20. «Bois et Chiffons si rares, si authentiques». Devise de l'enseigne qui regroupe, en 2002, une soixantaine de magasins implantés dans toute la France. Cette enseigne d'ameublement, d'arts de la table et de décoration " privilégie la mise en valeur et la mise en situation des articles dans un univers décoré et coloré ». Informations extraites du dossier de demande d'autorisation de création d'un magasin Bois et Chiffons de $815 \mathrm{~m}^{2}$ à la CDEC des Vosges, en octobre 2002.

21. Le CAPEV (Comité d'Aménagement, de Promotion et d'Expansion des Vosges), par sa connaissance du potentiel départemental, peut proposer un large éventail de conseils et d'aides pour la recherche d'un bâtiment ou d'un site industriel, pour la rencontre d'un partenaire financier ou industriel. 


\section{RÉSUMÉS}

Les zones d'activités industrielles et commerciales vosgiennes se concentrent très majoritairement dans les vallées de la Moselle et de la Meurthe. Près de la moitié de la superficie totale des zones est aménagée dans les deux agglomérations vosgiennes principales. Les zones industrielles représentent les quatre cinquièmes de l'ensemble de ces zones d'activités installées en périphérie urbaine. Elles se différencient d'une agglomération à l'autre par leur genèse (date de création, décideurs et acteurs) et leur occupation. Quant aux zones commerciales « d'entrée de ville", plus récentes, plus petites et se ressemblant davantage, elles sont également diversifiées. Nées parfois d'initiative privée, ce sont soit des concentrations de commerces entraînées par une locomotive incontestée (hypermarché), soit des concentrations de commerces de type «astéroïde " aménagées par les collectivités afin de tenter de lutter contre l'évasion commerciale. L'aménagement de ces périphéries urbaines n'est pas allé sans concurrences à l'intérieur des agglomérations ; ces concurrences pourraient s'atténuer à la faveur du développement de l'intercommunalité.

Industrial areas and the retail trade of the Vosges are largely concentrated in the Moselle and Meurthe valleys. Nearly half of the total area of these zones is developed in two principal cities of the Vosges. These industrial zones constitute four fifths of the activities of the peripheral areas of the cities. They are differentiated by origin (year of creation, initiator) and occupation. Retail trade areas at the edge of the cities are more recent, smaller and look more alike, though they are equally diversified. Sometimes created by private initiative, they are retail concentrations either attracted by an undisputed pacesetter (hypermarket), or of «asteroid» type developed by communities tempted to struggle against the flight of retailing. The development of these urban peripheries is not without competition from the interior of the cities; this competition could decrease with the development of the inter-communal cooperation.

Die Gewerbe- und Einzelhandelsparks der Vogesen konzentrieren sich hauptsächlich in den Tälern der Mosel und der Meurthe. Die Hälfte der Fläche der Parks befindet sich in den beiden Hauptverdichtungsräumen der Vogesen. Die Industrieparks entsprechen vier Fünfteln der Gewerbeparks an der Peripherie der Städte. Sie unterscheiden sich durch ihre Entstehung (Datum, Initiatoren und Träger des Projektes) und ihre Zusammensetzung. Die Einzelhandelsparks am Eingang der Städte sind kleiner, entstanden neuerdings und ähneln sich mehr. Sie entstanden manchmal infolge einer privaten Entscheidung und bestehen entweder aus Einzelhandelsgeschäften um ein Einkaufszentrum oder lediglich aus Einzelhandelsgeschäften, die von der Gemeindeverwaltung entwickelt wurden, um den Handelsverlust zu begrenzen. Die Entwicklung dieser peripheren Standorte führte $\mathrm{zu}$ einer Konkurrenz im Innern der Verdichtungsräume; mit der Entwicklung der interkommunalen Zusammenarbeit könnte diese Konkurrenz geringer werden.

\section{INDEX}

Keywords : city margin, collectivity, enterprise, industrial zone, retail trade, retail trade area, urban periphery, zone of activities

Schlüsselwörter : Aktivitätszone, Handelsinformation, Handelszone, Industriezone, Stadteingang, Städtische Peripherie, Territoriale Kollektivität, Unternehmen

Mots-clés : collectivité territoriale, enseigne commerciale, entrée de ville, entreprise, périphérie urbaine, zone commerciale, zone d'activités, zone industrielle 
AUTEUR

COLETTE RENARD-GRANDMONTAGNE

Université Nancy 2 - BP 33-97 - 54015 NANCY cedex 